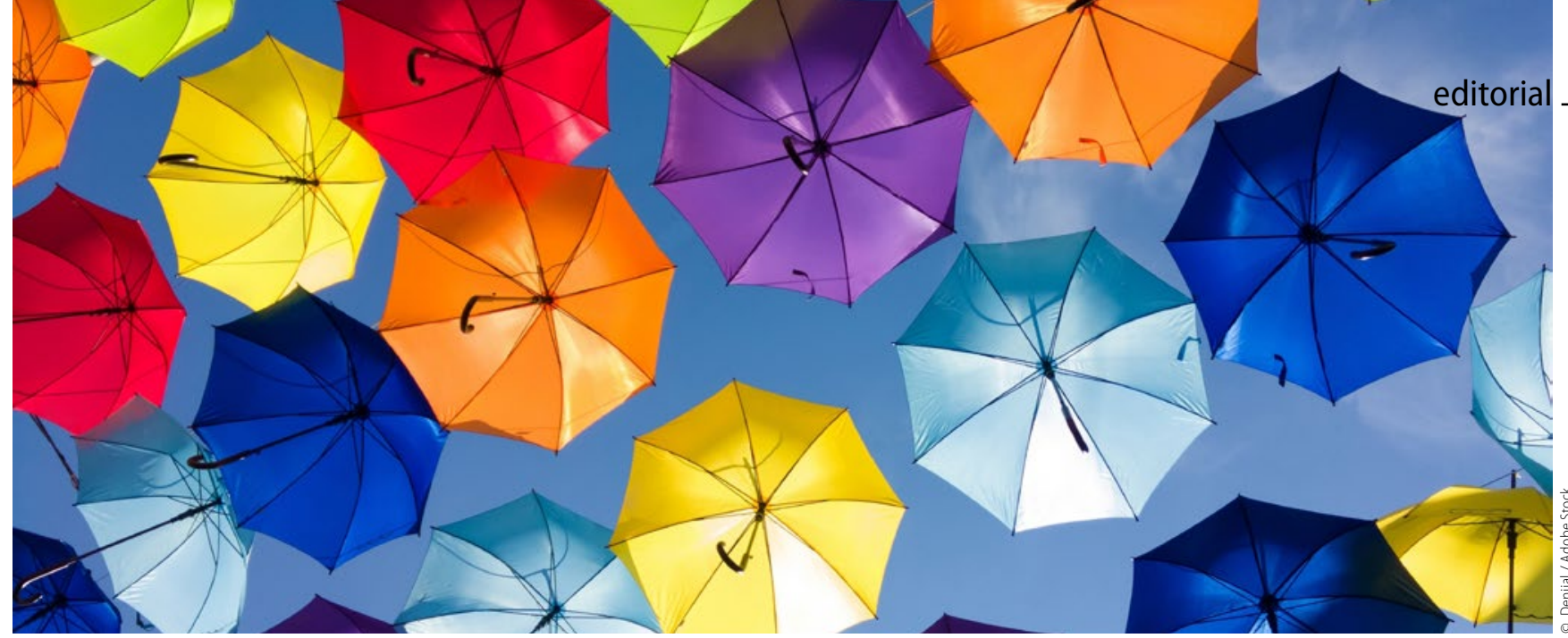

\title{
Beharrlichkeit zahlt sich aus - nicht alle Technologie ist böse
}

In dieser Ausgabe der InFo Neurologie \& Psychiatrie werden neue Daten zum Verschluss des persistierenden Foramen ovale (PFO) mit dem Ziel der Sekundärprävention nach Schlaganfall diskutiert (siehe Seite 10 ff.). Die neuen Daten sind bemerkenswert, zeigen sie doch, dass der „Schirmchenverschluss“ des PFO mittel- und langfristig die Zahl von Schlaganfallrezidiven effektiv senken kann. Vor wenigen Jahren, nach Erscheinen der ersten großen randomisierten, aber negativen Studie „CLOSURE I“ mit über 900 Patienten, haben sich viele von uns Neurologen bestätigt gefühlt, dass die Welt eben doch nicht so einfach, so „mechanisch“ ist. Das Motto „was zu ist, mach auf“ und „was auf ist, mach zu" behagt uns im Allgemeinen nicht, es wird der Komplexität der Neurologie nicht gerecht. Oder eben doch?

Manchmal doch. Denken wir einmal zurück: Die ersten Studien zur Operation von Stenosen der A. carotis interna waren negativ. Gescheitert. Heute ist diese Therapie internationaler Standard. Die ersten Studien zur katheterbasierten Therapie des akuten Schlaganfalls waren negativ. Gescheitert. Heute sind diese Therapien internationaler Standard. Wie beim PFO-Verschluss gab es nach den ersten negativen Studien genügend Neurologen, die höflich-analytisch, aber bestimmt konstatierten: „Seht Ihr, so einfach ist es eben nicht." Ich nehme mich da nicht aus. Ganz tief in unserem Inneren haben viele von uns aber gewusst, dass an der Mechanik etwas dran ist. Wie sonst könnte man erklären, dass auch nach den negativen Studien, viele „komplexe“ Einzelfallentscheidungen getroffen wurden, $\mathrm{PFO}$ zu verschließen, Karotiden zu erweitern oder in der Akutphase Katheterinterventionen einzusetzen. Forschere neurologische Kollegen waren beharrlich und haben sich gemeinsam mit Gefäßchirurgen, Neuroradiologen und Kardiologen in Folgestudien engagiert, die letztlich die entscheidende Evidenz für unser heutiges Handeln lieferten.

So weit, so gut. Kritisch, aber dann konstruktiv kooperierend. Allein die Tatsache, dass wir uns als Neurologen zu oft in Rückzugsgefechte begeben, sollten wir überdenken. „Nil nocere.“ Richtig. Aber das heißt nicht, dass wir „mechanisch“"bei Reflexhammer und Stimmgabel stehen bleiben sollten. Ich denke, man kann auch „kriminalistisch“ erfolgreich tätig sein, Rätsel lösen und hochkomplexe Zusammenhänge aufschlüsseln, ohne sich

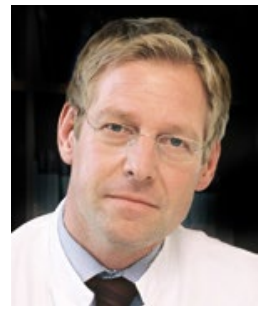

Prof. Dr. med. Christian Gerloff

Klinik und Poliklinik für Neurologie

Kopf- und Neurozentrum

Universitätsklinikum Hamburg-Eppendorf

Martinistr. 52, 20246 Hamburg

E-Mail: gerloff@uke.de

gegen mechanische Interventionen zu sträuben. Bei den genannten Beispielen war es die Beharrlichkeit kleiner Gruppen, oft von Nichtneurologen, die entscheidende Verbesserungen der Therapie bewirkt hat. Wir sollten uns an der Spitze solcher Innovationen bewegen, nicht in der Abwehr.

Es geht wohlgemerkt nicht um das unkritische Huldigen täglich neuer „Tools“. Es geht vielmehr darum, dass wir die Möglichkeiten neuer Technologien bereitwillig annehmen, uns aktiv einbringen und die klinische Neurologie damit noch interessanter machen. Wären wir proaktiver, wenn die Kathetertechniken in der Neurologie selbst verankert wären? Man könnte das denken; dagegen spricht aber, dass sich die Neurologie sukzessive selbst von den ihr ureigensten Technologien wie Elektrophysiologie und Neurosonografie entfernt und immer mehr ausschließlich ,in Bildern denkt“. Die Weiterbildungsassistenten bei der Facharztprüfung sind heute regelhaft fitter beim Interpretieren von MRT-Aufnahmen als von elektrophysiologischen Kurven und Werten.

Meine persönliche Sicht ist, dass minimal-invasive Techniken wie katheterbasierte Interventionen oder endoskopische Diagnostik und Therapie noch viele neue Wege in der Neurologie eröffnen können. Auch im Zeitalter erster funktionierender Gentherapien sind wir weit davon entfernt, solche Eingriffe zu den medizinhistorischen Akten zu legen. Ein bisschen mehr Technologieaffinität stünde uns nicht schlecht zu Gesicht. Ein Schelm, wer Böses dabei denkt.

Christian Gerloff 